\title{
Stereochemistry of atropisomeric 9,10-dihydrophenanthrene dimers from Pholidota chinensis
}

\author{
Sheng Yao, ${ }^{\mathrm{a}}$ Chun-Ping Tang, ${ }^{\mathrm{a}}$ Yang Ye, ${ }^{\mathrm{a}, *}$ Tibor Kurtán, ${ }^{\mathrm{b}}$ Attila Kiss-Szikszai, ${ }^{\mathrm{b}}$ \\ Sándor Antus, ${ }^{\mathrm{b}, \mathrm{c}}$ Gennaro Pescitelli, ${ }^{\mathrm{d}}$ Piero Salvadori, ${ }^{\mathrm{d}}$ and Karsten Krohn ${ }^{\mathrm{e}, *}$
}

${ }^{a}$ State Key Laboratory of Drug Research, Shanghai Institute of Materia Medica, Chinese Academy of Sciences, 555 Zu-Chong-Zhi Road, Zhangjiang Hi-Tech Park, Shanghai 201203, P. R. China

${ }^{b}$ Department of Organic Chemistry, University of Debrecen, P.O. Box 20, 4010 Debrecen, Hungary

${ }^{c}$ Research Group for Carbohydrates of the Hungarian Academy of Sciences, University of Debrecen, P.O.B. 55, H-4010 Debrecen, Hungary

${ }^{d}$ Dipartimento di Chimica e Chimica Industriale, Università di Pisa, via Risorgimento 35, I-56126 Pisa, Italy

${ }^{e}$ Department of Chemistry, University of Paderborn, Warburger Strasse 100, 33098 Paderborn, Germany

*Corresponding author. Tel.: +86 21 50806726; Fax: +86 21 50807088; e-mail: yye@ mail.shcnc.ac.cn (Y. Ye); Tel.: +49 525160 2172; Fax: +49 525160 3245; e-mail: k.krohn@upb.de (K. Krohn)

\begin{abstract}
Six bis-9,10-dihydrophenanthrene and 9,10-dihydrophenanthrene/(dihydro)stilbene derivatives, phochinenins G-L (1-6), were isolated from the whole plant of Pholidota chinensis. Their structures were elucidated on the basis of extensive spectroscopic investigations (1D, 2D NMR and HR-EIMS). Owing to the sterically hindered rotation around the biaryl axis, some of these biaryl compounds can exist as a pair of enantiomers, but were isolated as racemates. Computed inversion barriers of selected atropisomeric derivatives suggested that phochinenins $\mathrm{K}(\mathbf{5})$, gymconpin $\mathrm{C}(\mathbf{7})$ and flavanthrin (9) have optically stable atropisomers. Their racemates were separated by HPLC on an optically active stationary phase, and stereochemically characterized on-line by circular dichroism (CD) spectroscopy (LC-CD coupling), in conjunction with quantum-mechanics CD calculations.
\end{abstract}

Keywords: Pholidota chinensis; Phochinenins G-L; Dimeric 9,10-dihydrophenanthrene derivatives; Atropisomerism; Circular dichroism (CD); LC-CD coupling; CD calculations. 


\section{Introduction}

Dimeric 9,10-dihydrophenanthrene derivatives have frequently been isolated from the Orchidaceae family since the late eighties. ${ }^{1}$ Extracts from these plants contain various biaryl compounds including hetero- or homodimers, typically composed of a 9,10-dihydrophenanthrene or phenanthrene unit linked to a 9,10-dihydrophenanthrene, phenanthrene, stilbene, or dihydrostilbene unit, through $\mathrm{C}-\mathrm{C}$ or $\mathrm{C}-\mathrm{O}-\mathrm{C}$ bonds. ${ }^{2-6}$ Depending on the presence and nature of substituents on the ortho positions around the linked carbon atoms, the rotation around the biaryl axis may be not freely allowed due to steric hindrance. In such conditions, biaryls give rise to atropisomerism, ${ }^{7}$ i.e., they exist as pairs of optically stable enantiomers (atropisomers). This well-known phenomenon is still attracting attention in many fields such as natural products, ${ }^{8}$ molecular machines, ${ }^{9}$ and chemical synthesis. ${ }^{10}$ To the best of our knowledge, however, atropisomerism of natural biaryls from Orchidaceae has never been investigated thus far.

Pholidota chinensis (Orchidaceae) is a perennial herb distributed in Fujian, Guangdong, Guangxi and Yunnan provinces in China. ${ }^{11}$ From this plant six dimeric 9,10-dihydrophenanthrene derivatives, phochinenins A-F, were reported. ${ }^{12}$ In this paper, we describe the isolation and structural elucidation of nine biaryl compounds, phochinenins G-L (1-6), gymconpin $\mathrm{C}^{4}(\mathbf{7})$, blestrianol $\mathrm{A}^{2}(\mathbf{8})$ and flavanthrin ${ }^{1}$ (9). These compounds are formed by a 9,10-dihydrophenanthrene and a stilbene or a dihydrostilbene, or by two 9,10-dihydrophenanthrenes, through a direct C-C coupling. For some of these compounds, semi-empirical calculations suggested the possibility of occurrence of atropisomerism. Consequently, racemic 5, 7 and 9 were separated by analytical enantioselective HPLC into their stable atropisomers. A full stereochemical investigation was then carried out, in light of the poor attention received so far by chiral 9,10-dihydrophenanthrenes when compared to the corresponding fully unsatured bis-phenanthrenes. ${ }^{13}$ Therefore, we measured circular dichroism (CD) spectra of the separated enantiomers by employing the on-line HPLC-CD method, a useful technique for the characterization of enantiomers in mixtures of partially or fully separated stereoisomers. ${ }^{14,15}$ In this way, the absolute configuration of 9,10-dihydrophenanthrene dimers could be assigned by comparison of the CD spectra recorded on-line with the results of semi-empirical (NDO) CD calculations. 


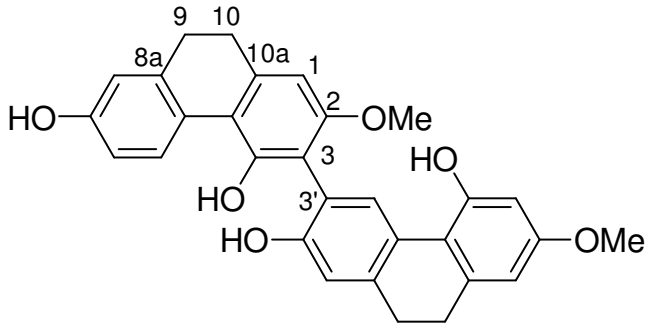

phochinenin $\mathrm{G}(\mathbf{1})$

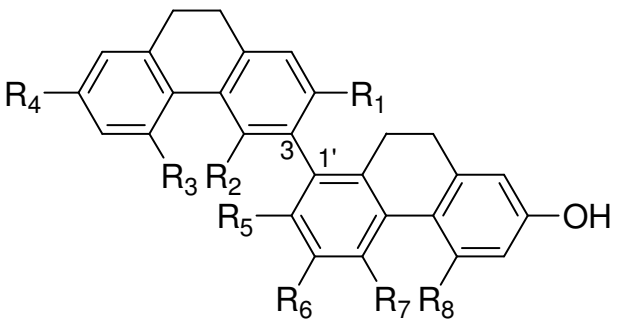

phochinenin $\mathrm{H}$ (2) $\mathrm{R}_{3}=\mathrm{R}_{5}=\mathrm{R}_{8}=\mathrm{H}, \mathrm{R}_{2}=\mathrm{R}_{4}=\mathrm{R}_{7}=\mathrm{OH}, \mathrm{R}_{1}=\mathrm{R}_{6}=\mathrm{OMe}$ gymconpin $C$ (7) $\mathrm{R}_{2}=\mathrm{R}_{6}=\mathrm{R}_{8}=\mathrm{H}, \mathrm{R}_{1}=\mathrm{R}_{4}=\mathrm{R}_{5}=\mathrm{OH}, \mathrm{R}_{3}=\mathrm{R}_{7}=\mathrm{OMe}$ blestrianol $A$ (8) $R_{3}=R_{6}=R_{8}=H, R_{1}=R_{4}=R_{5}=O H, R_{2}=R_{7}=O M e$<smiles>COc1cc(O)c2c(c1)CCc1cc(O)c(-c3c([14CH]Cc4cccc(O)c4)cc(O)cc3OC)cc1-2</smiles>

phochinenin I (3)

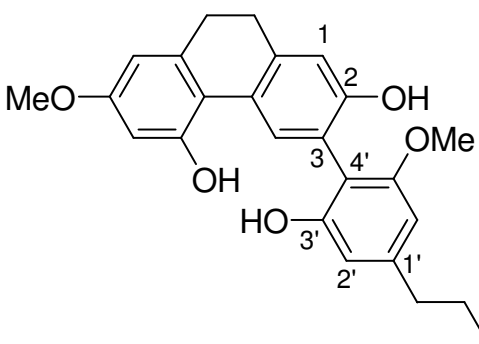

phochinenin J (4)

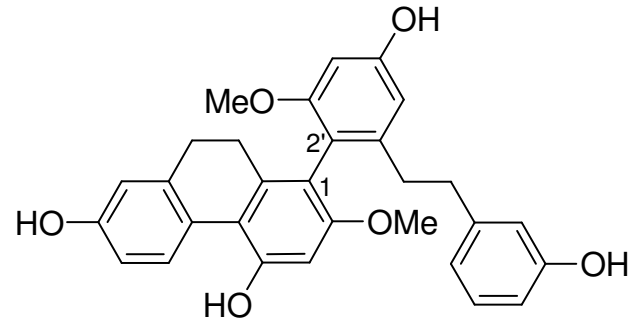

phochinenin $\mathrm{K}(\mathbf{5})$

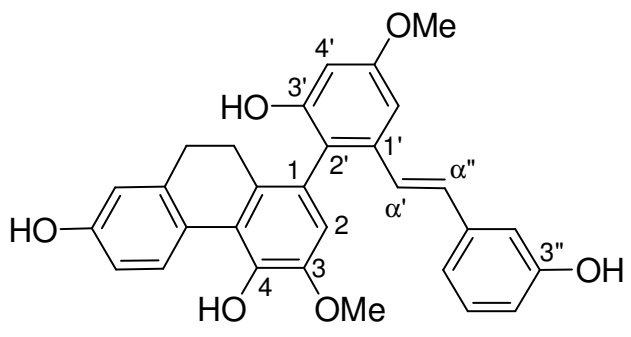

phochinenin L (6)

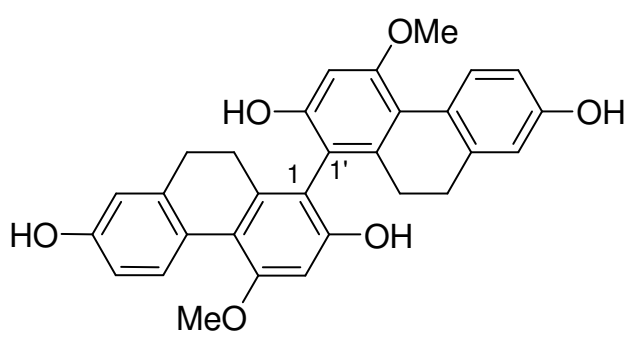

flavanthrin (9)

\section{Results and discussion}

\subsection{Structure elucidation}

Phochinenin G (1) was obtained as a brown amorphous powder. The molecular formula $\mathrm{C}_{30} \mathrm{H}_{26} \mathrm{O}_{6}$ was determined by HR-EIMS. The maximal UV absorptions at 213 and $279 \mathrm{~nm}$ indicated the presence of a 9,10-dihydrophenanthrene derivative. ${ }^{16}$ Analysis of ${ }^{1} \mathrm{H}$ and ${ }^{13} \mathrm{C}$-NMR spectra of $\mathbf{1}$ (Table 1) suggested an asymmetrical structure for a dimeric 9,10-dihydrophenanthrene derivative. The fragment ion at $\mathrm{m} / \mathrm{z}$ 241 in the EIMS spectrum indicated the same elemental composition $\mathrm{C}_{15} \mathrm{H}_{13} \mathrm{O}_{3}$ for the two monomeric halves. The HMBC correlations of H-10/C-1 and C-9, H-9/C-8, H-8/C-9, and H-1/C-10 indicated a 2,3,4,7-substituted 9,10-dihydrophenanthrene fragment. The other half was found to be substituted at C-2', $3^{\prime}, 5^{\prime}$ and $7^{\prime}$ by the HMBC correlations of $\mathrm{H}-10^{\prime} / \mathrm{C}-1^{\prime}$ and C-9', H-9'/C-8', and H-1'/C-10'. These 
two halves were coupled at C-3 and C-3' as demonstrated by the long correlations of H-1/C-3, H-1/C-3', $\mathrm{H}-1^{\prime} / \mathrm{C}-3^{\prime}, \mathrm{H}-1^{\prime} / \mathrm{C}-3$, and H-4'/C-3 (Figure 1). ROESY correlations of OMe $(\delta 3.75) / \mathrm{H}-1$, OMe $(\delta$ 3.77)/H-6' and H-8' allowed the two methoxys to be located at C-2 and C-7', respectively (Figure 1). The four hydroxyl groups, in turn, were attached to C-4, 7, 2' and 5', respectively. Therefore, 1 was established as 4,7,2',5'- tetrahydroxy-2,7'-dimethoxy-3,3'-bis-(9,10-dihydrophenanthrene).

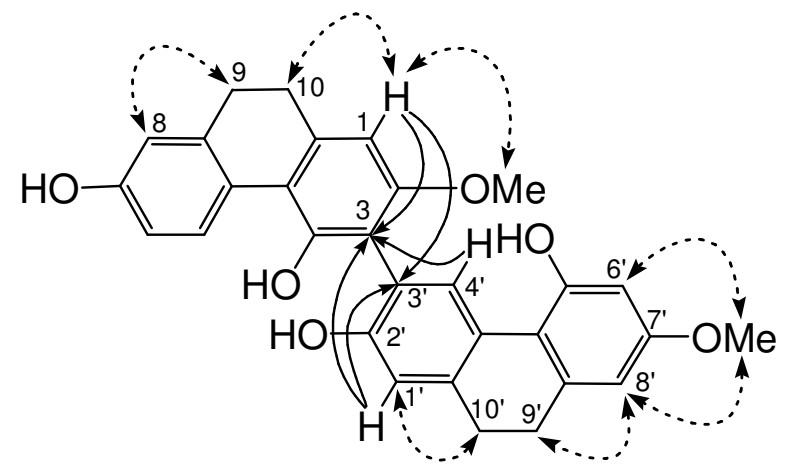

Figure 1. Key HMBC (arrows) and ROESY (broken arrows) correlations of $\mathbf{1 .}$

Phochinenin $\mathrm{H}$ (2) was obtained as a brown powder $\left(\mathrm{C}_{30} \mathrm{H}_{26} \mathrm{O}_{6}\right.$ by HR-EIMS). The UV spectrum showed characteristic absorptions for a 9,10-dihydrophenanthrene derivative at 211 and $282 \mathrm{~nm}$. Its NMR data (Table 1) indicated again a 9,10-dihydrophenanthrene dimer, which was composed of one 2,3,4,7-tetrasubstituted and one $1^{\prime}, 3^{\prime}, 4^{\prime}, 7^{\prime}$-tetrasubstituted moiety as deduced by a combined analysis of the HSQC, HMBC and ROESY spectra. The chemical shifts of four methylene signals at $\delta_{\mathrm{H}} 2.82(2 \mathrm{H}$, $m), 2.78(2 \mathrm{H}, m), 2.57(2 \mathrm{H}, m)$ and $2.39(2 \mathrm{H}, m)$ suggested that two of these methylenes $\left(\delta_{\mathrm{H}} 2.57\right.$ and 2.39) fall into the shielding zone of the neighboring aromatic ring and thus one monomer was substituted at C-1'. The other dimerization site was deduced to be $\mathrm{C}-3$ by HMBC correlations of H-10'/C-1', H-2'/C-1', H-2'/C-3and H-1/C-3. ROESY correlations of OMe $(\delta 3.68) / \mathrm{H}-1$, and $\mathrm{OMe}(\delta$ $3.88) / \mathrm{H}-2^{\prime}$ assigned two methoxys at $\mathrm{C}-2$ and $\mathrm{C}-3^{\prime}$, respectively.

Table 1. ${ }^{1} \mathrm{H}$ and ${ }^{13} \mathrm{C}$ NMR data of compounds $\mathbf{1}$ and $\mathbf{2}$ in $\mathrm{CD}_{3} \mathrm{OD}(\delta$ in ppm).

\begin{tabular}{|c|c|c|c|c|c|c|c|c|c|}
\hline & \multicolumn{2}{|c|}{$\delta_{\mathrm{H}}(600 \mathrm{MHz}, J$ in $\mathrm{Hz})$} & \multicolumn{2}{|c|}{$\delta_{\mathrm{C}}$} & & \multicolumn{2}{|c|}{$\delta_{\mathrm{H}}(600 \mathrm{HMz}, J$ in $\mathrm{Hz})$} & \multicolumn{2}{|c|}{$\delta_{\mathrm{C}}$} \\
\hline & 1 & 2 & $\mathbf{1}^{\mathrm{a}}$ & $2^{\mathrm{b}}$ & & 1 & 2 & $\mathbf{1}^{\mathrm{a}}$ & $2^{\mathrm{b}}$ \\
\hline 1 & $6.57 s$ & $6.57 s$ & 105.0 & 104.5 & $1^{\prime}$ & $6.84 s$ & & 116.4 & 111.8 \\
\hline 2 & & & 157.9 & 157.9 & $2^{\prime}$ & & $6.63 s$ & 154.7 & 99.7 \\
\hline 3 & & & 115.4 & 112.8 & $3^{\prime}$ & & & 119.1 & 159.3 \\
\hline 4 & & & 153.4 & 156.3 & $4^{\prime}$ & $8.15 \mathrm{~s}$ & & 133.8 & 153.5 \\
\hline $4 a$ & & & 141.2 & 141.2 & $4^{\prime} \mathrm{a}$ & & & 141.0 & 143.0 \\
\hline $4 \mathrm{~b}$ & & & 141.2 & 140.9 & $4^{\prime} \mathrm{b}$ & & & 142.4 & 141.3 \\
\hline
\end{tabular}




\begin{tabular}{|c|c|c|c|c|c|c|c|c|c|}
\hline 5 & $8.20 d(8.3)$ & $8.07 d(8.5)$ & 130.8 & 130.6 & $5^{\prime}$ & & $8.19 d(8.3)$ & 158.6 & 130.5 \\
\hline 6 & $\begin{array}{c}6.64 d d \\
(2.4,8.3)\end{array}$ & $6.66 d d(8.5,2.6)$ & 114.1 & 114.0 & $6^{\prime}$ & $\begin{array}{c}6.34 d \\
(2.4)\end{array}$ & $\begin{array}{c}6.64 d d \\
(8.3,2.7)\end{array}$ & 102.0 & 113.8 \\
\hline 7 & & & 156.7 & 156.4 & $7^{\prime}$ & & & 160.4 & 156.4 \\
\hline 8 & $6.65 d(2.4)$ & $6.69 d(2.6)$ & 115.6 & 115.4 & $8^{\prime}$ & $6.38 d(2.4)$ & $6.63 d(2.7)$ & 106.7 & 115.0 \\
\hline $8 \mathrm{a}$ & & & 127.1 & 126.9 & $8^{\prime} \mathrm{a}$ & & & 127.5 & 126.7 \\
\hline 9 & $2.75 \mathrm{~m}$ & $2.78 \mathrm{~m}$ & 31.5 & 31.6 & $9^{\prime}$ & $2.78 \mathrm{~s}$ & $2.57 \mathrm{~m}$ & 32.3 & 31.2 \\
\hline 10 & $2.79 \mathrm{~m}$ & $2.82 \mathrm{~m}$ & 32.5 & 32.4 & $10^{\prime}$ & $2.78 \mathrm{~s}$ & $2.39 \mathrm{~m}$ & 31.2 & 28.6 \\
\hline $10 \mathrm{a}$ & & & 117.5 & 117.0 & $10^{\prime} \mathrm{a}$ & & & 116.6 & 118.2 \\
\hline \multirow[t]{2}{*}{ 2-OMe } & $3.75 s$ & $3.68 s$ & 56.0 & 56.2 & 7'-OMe & $3.77 s$ & & 56.0 & \\
\hline & & & & & 3'-OMe & & $3.88 s$ & & 56.4 \\
\hline
\end{tabular}

${ }^{\mathrm{a}}$ At $100 \mathrm{MHz},{ }^{\mathrm{b}}$ At $125 \mathrm{MHz}$

The molecular formula of phochinenin I (3) was determined to be $\mathrm{C}_{30} \mathrm{H}_{28} \mathrm{O}_{6}$ by HR-EIMS. UV absorption maxima were observed at 201 and $279 \mathrm{~nm}$. Its NMR (Table 2) showed signals for a 2,3,5,7-tetrasubsituted 9,10-dihydrophenanthrene and signals for a $2^{\prime}, 3^{\prime}, 5^{\prime}, 3^{\prime \prime}$-tetrasubstituted dihydrostilbene, indicating 3 to be a formed by a 9,10-dihydrophenanthrene and a dihydrostilbene. The linkage between C-3 and C-2' was revealed by HMBC correlations of H-4/C-3, H-4/C-2'and H- $\alpha^{\prime} / \mathrm{C}-2^{\prime}$ (Figure 2). Two methoxy groups were assigned at C-7 and C-3' by the ROESY experiment (Figure 2).

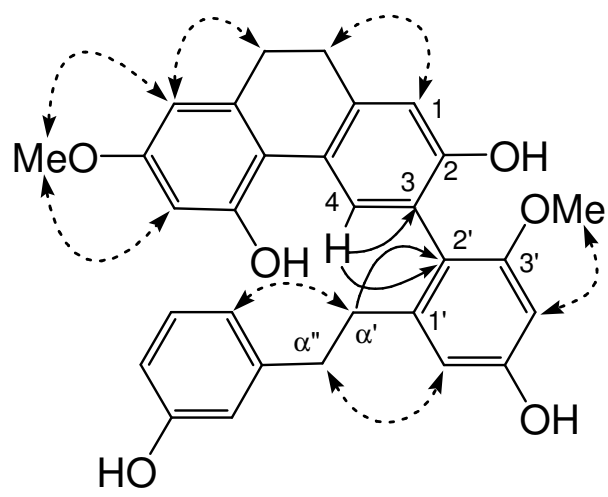

Figure 2. Key HMBC (arrows) and ROESY (broken arrows) correlations of $\mathbf{3}$.

The molecular formulae of phochinenins $\mathrm{J}(\mathbf{4})$ and $\mathrm{K}(\mathbf{5})$ were established by HR-EIMS as $\mathrm{C}_{30} \mathrm{H}_{28} \mathrm{O}_{6}$, the same as that of $\mathbf{3}$. Their UV and NMR spectra (Table 2), similar to those of $\mathbf{3}$, indicated that both $\mathbf{4}$ and 5 were composed of a 9,10-dihydrophenanthrene and a dihydrostilbene. The spectral differences were indicative of the dimerization sites and substitution patterns. The two halves of $\mathbf{4}$ were found to be coupled at C-3 and C-4' by long-range correlations of H-4/C-4', H-2'/C-4' and H-6'/C-4'. Compound 5 was determined to be a 1, 2'-coupled dimer, which was demonstrated by the upfield shifts of four typical methylenes in the structure. The substitution patterns were assigned by the ROESY spectrum. 
Phochinenin L (6) was obtained as a brown amorphous powder; its formula was established as $\mathrm{C}_{30} \mathrm{H}_{26} \mathrm{O}_{6}$ by HR-EIMS. UV absorption maxima appeared at 212, 281 and $297 \mathrm{~nm}$. The ${ }^{1} \mathrm{H}-\mathrm{NMR}$ spectrum of 6 (Table 2) was similar to those of compounds 3-5 except for a pair of trans-configured olefinic protons at $\delta_{\mathrm{H}} 6.78(1 \mathrm{H}, d, J=16.0 \mathrm{~Hz})$ and $6.97(1 \mathrm{H}, d, J=16.0 \mathrm{~Hz})$, corresponding to two olefinic methines $\left(\delta_{\mathrm{C}} 130.9\right.$ and 128.6) in its ${ }^{13} \mathrm{C}$-NMR spectrum (Table 2). This proved that instead of dihydrostilbene, a stilbene moiety was present in this molecule. A detailed analysis of its NMR data revealed that 6 consisted of a 1,3,4,7-tetrasubstituted 9,10-dihydrophenanthrene and a $2^{\prime}, 3^{\prime}, 5^{\prime}, 3^{\prime \prime}$-tetrasubstituted stilbene. HMBC correlations of H-10/C-1, H-2/C-1, H-2/C-2' and H- $\alpha^{\prime} / \mathrm{C}-2^{\prime}$ indicated that these two fragments were linked at C-1 and C-2'. Two methoxy groups were attached to C-3 and C-5', respectively, as proved by ROESY correlations of OMe $\left(\delta_{\mathrm{H}} 3.89\right) / \mathrm{H}-2$ and $\mathrm{H}-8$ and $\mathrm{OMe}$ $\left(\delta_{\mathrm{H}} 3.94\right) / \mathrm{H}-4^{\prime}$ and $\mathrm{H}-6^{\prime}$.

Table 2. ${ }^{1} \mathrm{H}$ and ${ }^{13} \mathrm{C}$ NMR data of compounds 3-6 (in $\left.\mathrm{MeOH}-d_{4}\right)(\delta$ in ppm).

\begin{tabular}{|c|c|c|c|c|c|c|c|c|}
\hline & \multicolumn{4}{|c|}{$\delta_{\mathrm{H}}(300 \mathrm{HMz}, J$ in $\mathrm{Hz})$} & \multicolumn{4}{|c|}{$\delta_{\mathrm{C}}$} \\
\hline & 3 & 4 & 5 & 6 & $3^{\mathrm{a}}$ & $4^{b}$ & $5^{b}$ & $6^{b}$ \\
\hline 1 & $6.81 \mathrm{~s}$ & $6.77 s$ & & & 115.8 & 116.0 & 115.9 & 115.3 \\
\hline 2 & & & & $6.63 s$ & 154.2 & 154.0 & 159.0 & 99.5 \\
\hline 3 & & & $6.59 s$ & & 119.6 & 116.7 & 99.8 & 159.1 \\
\hline 4 & $8.10 s$ & $8.07 \mathrm{~s}$ & & $8.04 s$ & 133.4 & 133.4 & 155.9 & 156.0 \\
\hline $4 a$ & & & & & 140.2 & 139.9 & 142.1 & 141.3 \\
\hline $4 \mathrm{~b}$ & & & & & 142.2 & 142.2 & 141.2 & 142.3 \\
\hline 5 & & & $8.03 d(8.5)$ & $8.10 d(8.6)$ & 157.1 & 156.0 & 130.8 & 130.8 \\
\hline 6 & $6.31 d(2.7)$ & $6.31 d(2.6)$ & $\begin{array}{c}6.62 d d(2.7 \\
8.5)\end{array}$ & $\begin{array}{c}6.66 d d(2.5 \\
8.6)\end{array}$ & 101.8 & 101.9 & 114.1 & 113.9 \\
\hline 7 & & & & & 161.4 & 160.1 & 156.5 & 156.6 \\
\hline 8 & $6.35 d(2.7)$ & $6.34 d(2.6)$ & $6.58 d(2.7)$ & $6.60 d(2.5)$ & 108.0 & 106.5 & 115.2 & 115.2 \\
\hline $8 \mathrm{a}$ & & & & & 122.1 & 120.1 & 118.0 & 117.8 \\
\hline 9 & $2.75 s$ & $2.71 s$ & $2.52 \mathrm{~m}$ & $2.47 \mathrm{~m}$ & 32.3 & 32.3 & 31.5 & 31.5 \\
\hline 10 & $2.75 s$ & $2.71 s$ & $2.33 m$ & $2.28 m$ & 31.3 & 31.2 & 28.8 & 28.8 \\
\hline $10 \mathrm{a}$ & & & & & 127.0 & 126.8 & 127.0 & 127.0 \\
\hline $1^{\prime}$ & & & & & 144.9 & 144.5 & 145.2 & 140.9 \\
\hline $2^{\prime}$ & & $6.41 d(1.1)$ & & & 116.6 & 110.3 & 117.3 & 118.0 \\
\hline $3^{\prime}$ & & & & & 160.1 & 156.7 & 161.8 & 158.1 \\
\hline $4^{\prime}$ & $6.33 d(2.4)$ & & $6.34 d(2.5)$ & $6.48 d(2.6)$ & 100.5 & 114.1 & 100.6 & 102.4 \\
\hline $5^{\prime}$ & & & & & 156.7 & 160.0 & 157.9 & 162.0 \\
\hline $6^{\prime}$ & $6.34 d(2.4)$ & $6.45 d(1.1)$ & $6.36 d(2.5)$ & $6.94 d(2.6)$ & 106.4 & 105.2 & 107.9 & 103.0 \\
\hline$\alpha^{\prime}$ & $2.60 m$ & $2.86 \mathrm{~m}$ & $2.54 m$ & $6.78 d(16.0)$ & 38.2 & 39.5 & 38.4 & 128.6 \\
\hline$\alpha^{\prime \prime}$ & $2.67 \mathrm{~m}$ & $2.86 \mathrm{~m}$ & $2.56 \mathrm{~m}$ & $6.97 d(16.0)$ & 38.5 & 39.1 & 38.5 & 130.9 \\
\hline $1 "$ & & & & & 145.7 & 145.1 & 145.7 & 140.6 \\
\hline $2 "$ & $\begin{array}{c}6.40 d d(2.4 \\
2.6)\end{array}$ & $\begin{array}{c}6.68 d d(2.1 \\
1.7)\end{array}$ & $\begin{array}{c}6.38 d d(2.1 \\
2.1)\end{array}$ & $\begin{array}{c}6.72 d d(2.1 \\
2.1)\end{array}$ & 116.7 & 116.7 & 116.7 & 116.2 \\
\hline
\end{tabular}




\begin{tabular}{|c|c|c|c|c|c|c|c|c|}
\hline 3" & & & & & 158.3 & 158.7 & 158.6 & 159.0 \\
\hline 4" & $\begin{array}{c}6.47 d d(2.4 \\
8.0)\end{array}$ & $\begin{array}{c}6.60 d d(7.9 \\
1.7)\end{array}$ & $\begin{array}{c}6.49 d d(2.1 \\
8.2)\end{array}$ & $\begin{array}{c}6.62 d d(2.1 \\
7.7)\end{array}$ & 113.7 & 114.1 & 114.0 & 113.7 \\
\hline 5" & $\begin{array}{c}6.93 d d(7.4 \\
8.0)\end{array}$ & $\begin{array}{c}7.09 d d(7.9 \\
7.5)\end{array}$ & $\begin{array}{c}6.94 d d(8.2 \\
8.6)\end{array}$ & $\begin{array}{c}7.07 d d(7.7 \\
8.1)\end{array}$ & 130.3 & 130.6 & 130.6 & 131.1 \\
\hline 6" & $\begin{array}{c}6.39 d d(2.6 \\
7.4)\end{array}$ & $\begin{array}{c}6.72 d d(7.5 \\
2.1)\end{array}$ & $\begin{array}{c}6.37 d d(2.1 \\
8.6)\end{array}$ & $\begin{array}{c}6.86 d d(8.1 \\
2.1)\end{array}$ & 121.3 & 121.2 & 121.3 & 120.1 \\
\hline 2-OMe & & & $3.89 \mathrm{~s}$ & & & & 56.5 & \\
\hline 3-OMe & & & & $3.89 \mathrm{~s}$ & & & & 56.3 \\
\hline 7-OMe & $3.77 s$ & $3.74 s$ & & & 55.9 & 55.6 & & \\
\hline 3'-OMe & $3.76 s$ & $3.67 \mathrm{~s}$ & $3.74 s$ & & 55.8 & 55.8 & 56.0 & \\
\hline \multicolumn{9}{|l|}{ 4'-OMe } \\
\hline 5'-OMe & & & & $3.94 s$ & & & & 56.2 \\
\hline At $100 \mathrm{~N}$ & At $125 \mathrm{M}$ & & & & & & & \\
\hline
\end{tabular}

In addition to the six new compounds, three other known dimers, gymconpin $\mathrm{C}^{4}(\mathbf{7})$, blestrianol $\mathrm{A}^{2}(\mathbf{8})$ and flavanthrin ${ }^{1}(\mathbf{9})$, were also isolated from $P$. chinensis. Their structures were determined by comparison of NMR and MS data with those reported in the literature.

\subsection{Stereochemistry assignment of atropisomeric biaryls}

As discussed in the Introduction, some of the isolated biaryl compounds may in principle exist as pair of optically-stable atropisomers. ${ }^{7}$ However, in the current case, optical rotations of all compounds were zero or negligible indicating that they were isolated as racemic mixtures of atropisomers, wherever atropisomerism was feasible. In order to reveal the presence of hindered rotation, the inversion barriers of selected dimers were calculated with the procedure described in the Computational Section. The semi-empirical PM3 method ${ }^{17}$ was employed, which has revealed to be especially accurate in the prediction of torsional energies and barriers. ${ }^{18}$ Table 3 reports PM3-computed inversion barriers for compounds 3-5, 7 and 9, having different biaryl skeletons and substitution patterns. Except for 4, inversion barriers around or above $20 \mathrm{kcal} / \mathrm{mol}$ were found, suggesting the possible existence of atropisomerism. ${ }^{7}$ Based on these results, three compounds $(\mathbf{5}, \mathbf{7}$ and $\mathbf{9})$ were selected for the separation of their atropisomers on an optically active stationary phase of analytical HPLC. In accordance with the calculations, the atropisomers of $\mathbf{5 , 7}$ and $\mathbf{9}$ could be base-line separated on a Chiralcel IA column after optimization of the conditions (see Experimental Section). Compounds $\mathbf{7}$ and $\mathbf{9}$ are 9,10-dihydrophenanthrene dimers that differ in the biaryl linkage; $\mathbf{9}$ is a symmetrical dimer, while $\mathbf{7}$ is unsymmetrical. These two compounds were studied by HPLC in their mixture containing 9 as the major component. Both the atropisomers of $\mathbf{7}$ and $\mathbf{9}$ could be base-line separated on Chiralcel IA 
column and they could be identified on the basis of the LC/CD-LC/UV chromatogram monitored at $270 \mathrm{~nm}$ (Figure 3).

Table 3. PM3-computed inversion barriers for selected compounds (in $\mathrm{kcal} / \mathrm{mol}$ ). ${ }^{\mathrm{a}}$

\begin{tabular}{cccccc}
\hline Compound & $\mathbf{3}^{\mathrm{b}}$ & $\mathbf{4}^{\mathrm{b}}$ & $\mathbf{5}^{\mathrm{b}}$ & $\mathbf{7}$ & $\mathbf{9}$ \\
Barrier & 19.5 & 12.5 & 30.0 & 20.0 & 25.0 \\
\hline
\end{tabular}

${ }^{\text {a }}$ See Computational Section for details.

${ }^{\mathrm{b}}$ Calculated on a reduced model (see Computational Section).

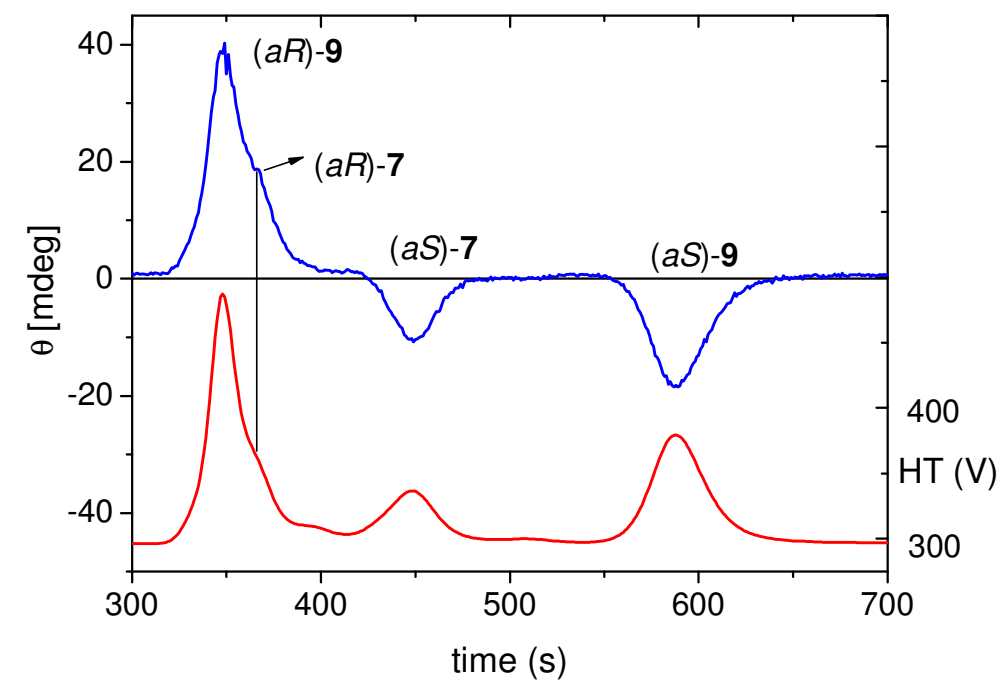

Figure 3. LC/CD (upper curve) and LC/UV (lower curve) chromatograms of a sample containing ( \pm )-7 and ( \pm )-9 monitored at $270 \mathrm{~nm}$. The high tension (HT) recorded directly in voltage has logarithmic relationship with the concentration or absorbance and it was not converted. For the chromatographic protocol, see the Experimental Section.

By stopping the flow at the UV/CD maxima, mirror image LC/CD spectra of the corresponding atropisomers of 7 and 9 could be recorded (Figures 4 and 5) which also enabled their configurational assignments by comparison with calculated CD spectra, as discussed below. The second and third eluted peaks of the chromatogram in Figure 3 showed mirror image CD spectra of $(a R)-7$ and $(a S)-7$, respectively, with four bands of alternating sign which can be interpreted as two oppositely signed exciton couplets ${ }^{19}$ centered around 281 and $232 \mathrm{~nm}$ (Figure 4). Similarly, the first and fourth eluted peaks belong to the $(\mathrm{a} R)$ and $(\mathrm{a} S)$ atropisomers of 9 , respectively, and showed opposite CD couplets 
(Figure 5).

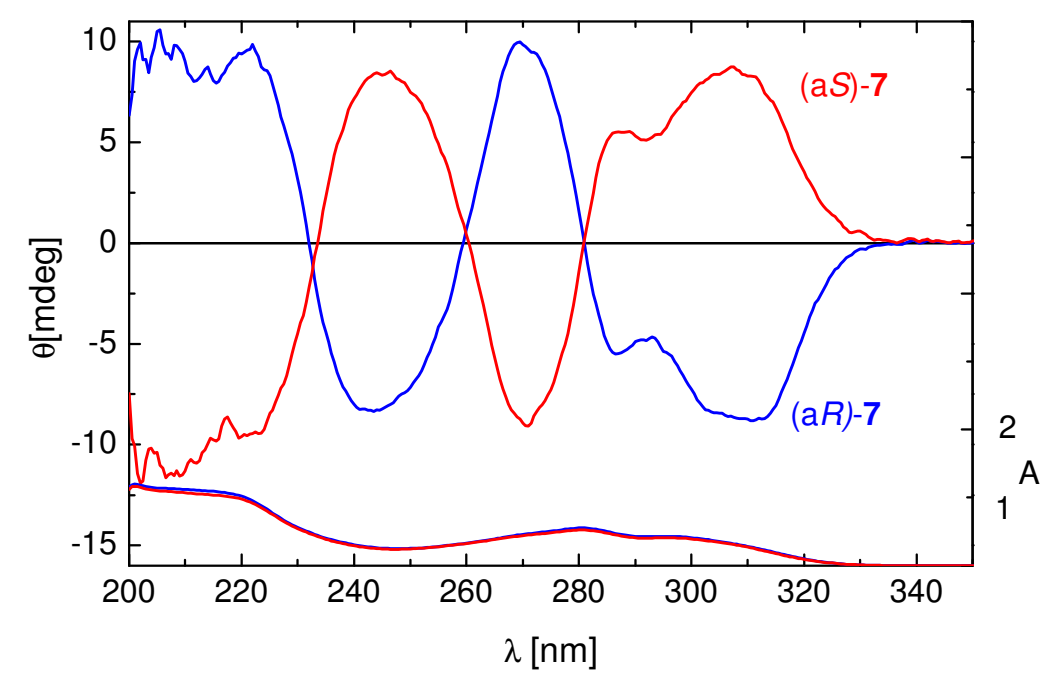

Figure 4. CD and UV spectra of (aS)-7 (red line) and (aR)-7 (blue line) in hexane/ethanol, 7:3.

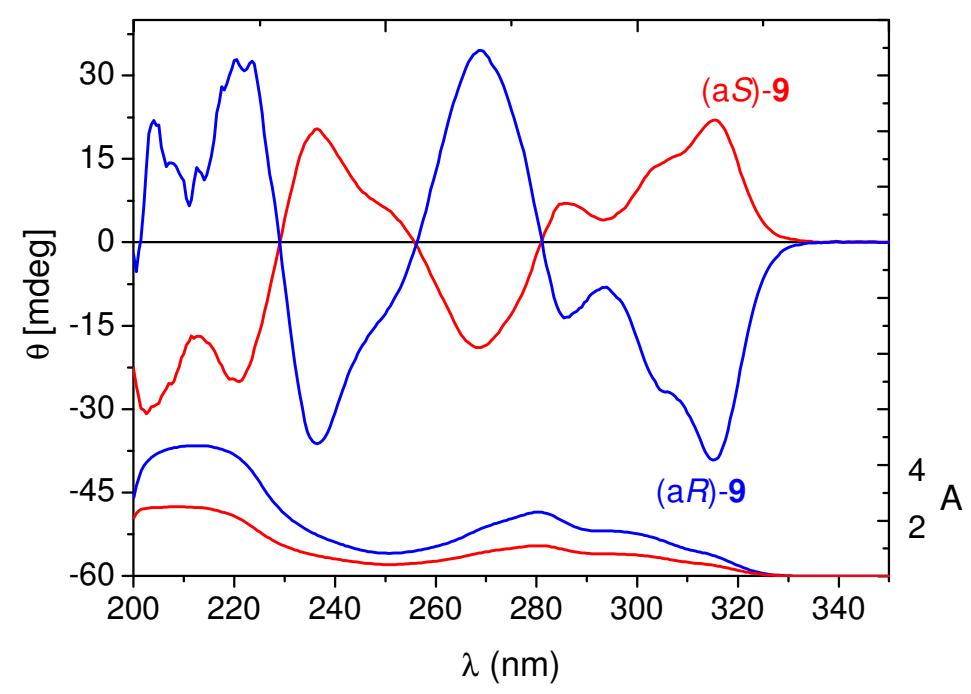

Figure 5. CD and UV spectra of (aS)-9 (red line) and (aR)-9 (blue line) in hexane/ethanol, 7:3.

The atropisomers of compound $\mathbf{5}$, constructed from a dihydrophenanthrene and a dihydrostilbene unit, gave significantly different $C D$ from those of dimers 7 and 9. In the $330-270 \mathrm{~nm}$ range, the CD spectrum is dominated by the transitions of the dihydrophenanthrene chromophore and strong exciton couplets could not be clearly identified (Figure 6). 


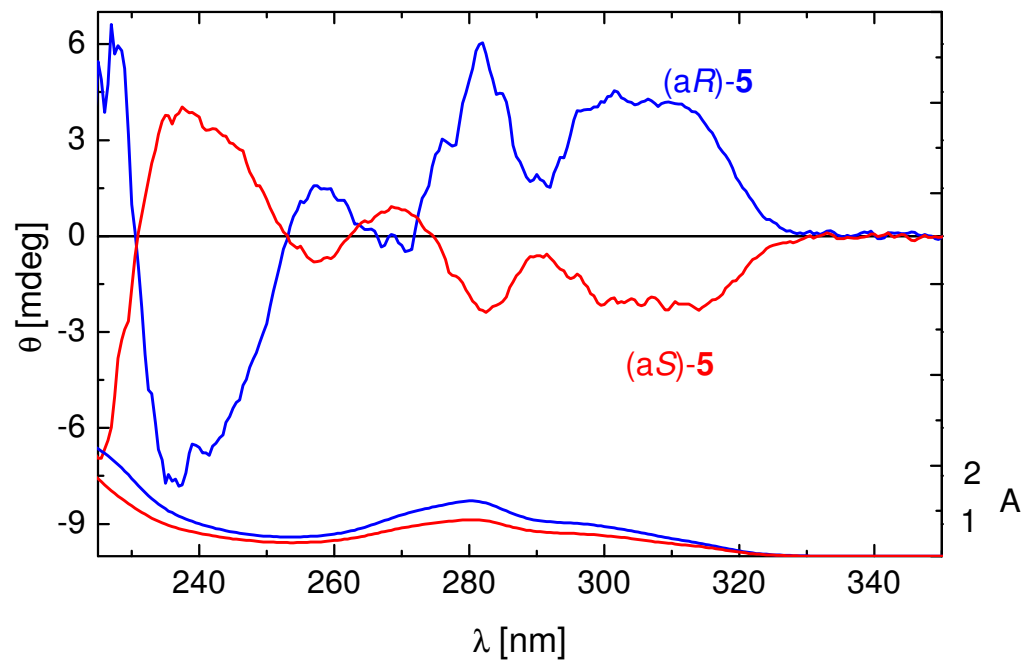

Figure 6. CD and UV spectra of (aS)-5 (red line) and (aR)-5 (blue line) in hexane/ethanol, 7:3.

As noticed in the Introduction, no CD data are reported for compounds similar to ours which could serve as reference for the stereochemical assignment. The CD spectra of 5, 7 and $\mathbf{9}$ may in principle derive from a combination of exciton coupling ${ }^{19}$ between strong dipole-allowed transitions of the aromatic chromophores (9,10-dihydrophenanthrene and benzene substituted with oxygenated groups) and other mechanisms of optical activity including the inherent chirality of each 9,10-dihydrophenanthrene moiety, ${ }^{20}$ equivalent to a twisted biphenyl with a small dihedral angle ( $\pm 27-28$ deg, from DFT structures). Each 9,10-dihydrophenanthrene unit may be thought to rapidly interconvert at room temperature in solution between $M$ and $P$ enantiomorphous arrangements. This element of chirality combines with the main atropisomerism discussed so far, thus generating in principle diastereomeric pairs which may have different energies and contributions to the overall CD. In these conditions, a straightforward application of the exciton chirality method ${ }^{18}$ is precluded. Therefore we decided to run semi-empirical quantum-mechanics CD calculations with the ZINDO method, ${ }^{21,22}$ using DFT-optimized geometries as input structures. For compounds 5, 7 and 9, a limited ensemble of 2-4 structures was generated with PM3 geometry optimizations (see Computational Section), refined with DFT optimizations, and employed for ZINDO-S/CI calculations. Additionally, the excited states of 1,3,6-trihydroxy-9,10-dihydrophenanthrene (10, Figure 7) were also calculated with ZINDO-S/CI, taken as a chromophoric model of the 9,10-dihydrophenanthrene unit found in the compounds considered. 


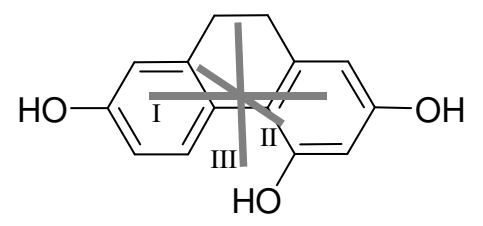

10

Figure 7. The chromophoric model of the 9,10-dihydrophenanthrene unit, showing the polarization of the main transitions computed by ZINDO (I, 312 nm; II, 305 nm; III, $240 \mathrm{~nm}$ )

Figure 8a-c reports ZINDO-calculated CD spectra on the various structures for each compound 5, 7 and 9, and the respective Boltzmann-weighted averages at room temperature according to DFT energies. Two average spectra are shown for each compound, obtained by employing different Gaussian band-widths ( $\sigma=2500$ and $4200 \mathrm{~cm}^{-1}$ half-height widths). For (aS)-7 and (aS)-9, a positive couplet-like $\mathrm{CD}$ feature is observed between 300 and $350 \mathrm{~nm}$, arising from the superimposition of four CD signals with alternating signs $+/-/+/-$ (from the right to the left) in this region. They are seemingly due to the exciton coupling between first two transitions of the monomer 10, the first of which (dipole I in Figure 7 ) is the strongest one and polarized along its long axis. The two couplets are both positive for the (aS) configuration, corresponding to biaryl $P$ helicity and positive chirality between the long axes of dihydrophenanthrenes (Figure 8d), in agreement with the expectations based on exciton chirality. ${ }^{19}$ Therefore, the sign of the experimental CD couplet observed for 7 and 9 in the $260-330 \mathrm{~nm}$ region can be taken as a proof of the absolute configuration, with the following correspondence: $(+)$ couplet $\leftrightarrow P$ chirality $\leftrightarrow(\mathrm{a} S)$ configuration. The CD band following the first couplet, with the same sign of the longer-wavelength branch, is found experimentally around $240 \mathrm{~nm}$ (Figures 4 and 5). One of its contributions (possibly the strongest one) is from an intrinsically CD-active biphenyl-like transition, ${ }^{20}$ computed for the monomer $\mathbf{1 0}$ at $240 \mathrm{~nm}$ (dipole III in Figure 9), with moderate rotational strength and sign depending on the twist between the two phenyl rings in the dihydrophenanthrene moiety. Therefore, it varies substantially among the four conformers 1-4 for each compound (7 and 9), and the apparent $\mathrm{CD}$ arises from an average of sizeably different contributions. Nonetheless, the dominant sign is positive for (aS) configuration, in keeping with experimental results (relatively to the long-wavelength couplet sign). In the shorter wavelength region, the calculated CD becomes less accurate, especially due to the superimposition of several transitions, although the sign of the dominant around $220 \mathrm{~nm}$ band seems also to be well predicted. The above results demonstrate that while the long-wavelength region (above $260 \mathrm{~nm}$ ) in the CD spectra of 7 and 9 may be fully interpreted according to the exciton theory, the opposite is true for the short-wavelength region; thus, our 
calculation approach is justified.

For compound 5, the coupling between a 1,3,6-trihydroxy-9,10-dihydrophenanthrene and a 2,6-dihydroxybenzene chromophore is observed. As a consequence, the CD is weaker and its interpretation less straightforward. However, the computed average CD (Figure 8c) is again in good agreement with the experimental one (Figure 6) and demonstrates the following correspondence: (-) bands at 270-320 $\mathrm{nm} \leftrightarrow(\mathrm{aS})$ configuration. By comparison between experimental and calculated spectra (Figure 8a-c vs. 4-6), we conclude that the second eluted enantiomers for each compound 5, 7 and $\mathbf{9}$, has (aS) configuration.
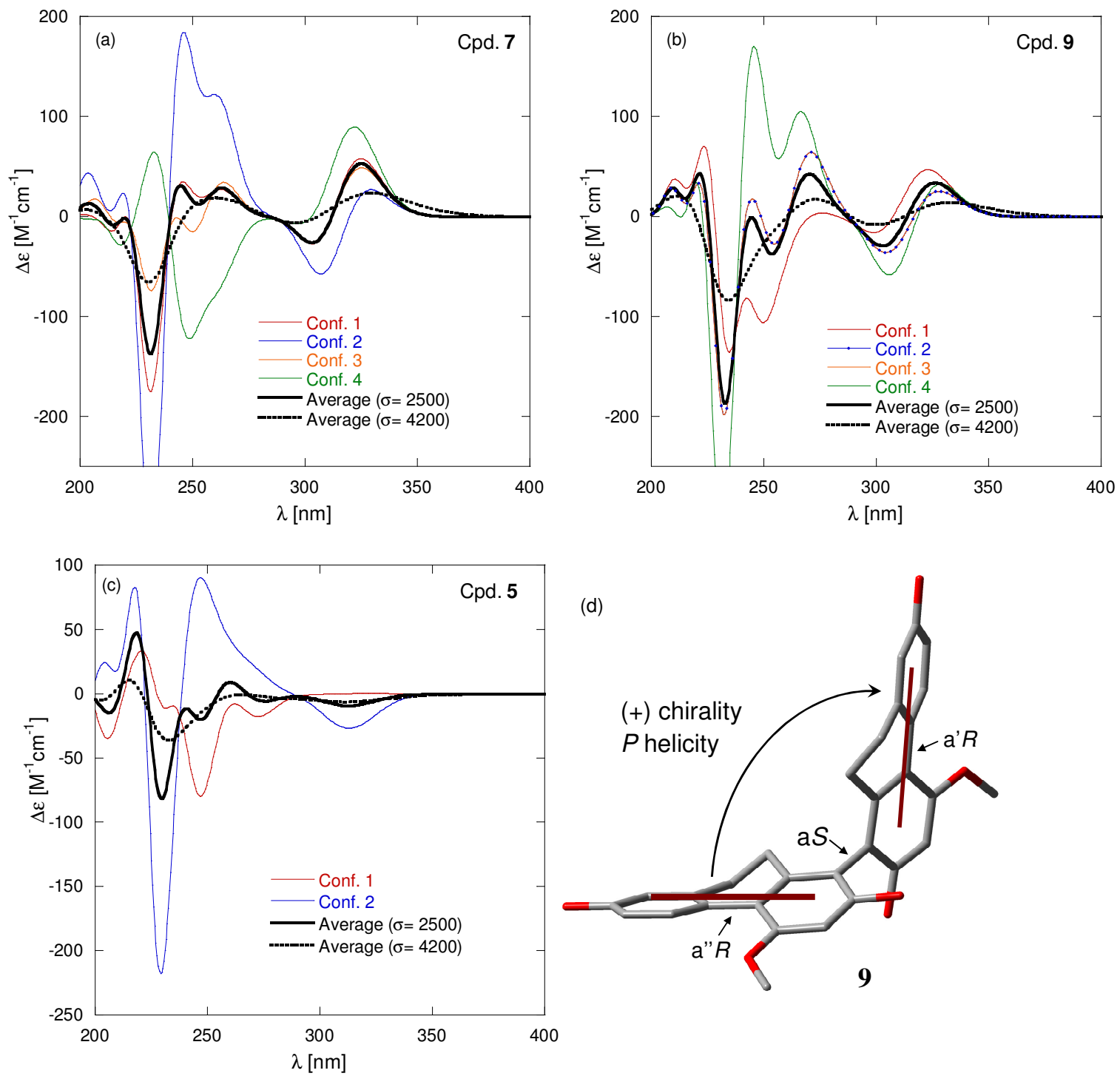

Figure 8. (a-c) ZINDO-calculated CD spectra of compounds 5, 7 and 9, for DFT-optimized structures, and their average (thick lines) computed with two Gaussian half-height widths ( $\sigma=2500$ and 4200 
$\mathrm{cm}^{-1}$ ); see Computational Section for details. (d) Lowest-energy DFT structure for compound $\mathbf{9}$, showing the $P$-helicity arrangement of dihydrophenanthrene units and long-axis polarized transition dipoles.

\section{Conclusions}

In this paper, the isolation and stereochemistry of nine naturally-occurring atropisomeric 9,10-dihydrophenanthrene derivatives is reported. These axially-chiral compounds were isolated as racemates from the plant and the absolute stereochemistry of the separated enantiomers was discussed here for the first time. The HPLC-CD spectra of selected atropisomers were recorded upon their separation on a chiral stationary phase. By comparison with the CD spectra calculated with ZINDO method, the absolute configuration of each enantiomer could be assigned.

According to the results of quantum-mechanics calculations, for compounds $\mathbf{7}$ and $\mathbf{9}$, the sign of the experimental CD couplet in the $260-330 \mathrm{~nm}$ region was correlated with the absolute configuration, with the correspondence: $(+)$ couplet $\leftrightarrow(\mathrm{a} S)$ configuration. For 5, a similar correspondence exists: $(-)$-CD maxima in the $270-320 \mathrm{~nm}$ region $\leftrightarrow(\mathrm{a} S)$ configuration

\section{Experimental}

\subsection{General experimental procedures}

Optical rotations were measured with a Perkin-Elmer 241MC polarimeter or a Perkin-Elmer 341 polarimeter. UV spectra were recorded with a Beckman DU-7 spectrometer. IR spectra were recorded using a Perkin-Elmer 577 Spectrometer. LR-EIMS were obtained on a MAT-95 spectrometer, HR-EIMS were obtained on a Kratos 1H spectrometer. NMR spectra were run on a Bruker AM-400, AM-500 or a Bruker AM-600 spectrometer with TMS as internal standard. Column chromatographic separations were carried out by using silica gel H60 (300-400 mesh, Qingdao Haiyang Chemical Group Corporation, People's Republic of China), MCI GEL CHP20P (75-150 $\mu \mathrm{m}$, Mitsubishi Chemical Industries) and Sephadex LH-20 (Pharmcia Biotech AB, Uppsala, Sweden) as packing materials. HSGF $_{254}$ silica gel TLC plates (Yantai Chemical Industrial Institute, People's Republic of China) were used for analytical TLC, and Pre-TLC plates $(20 \times 20 \mathrm{~cm}, 0.4 \sim 0.5 \mathrm{~mm}$, Yantai Chemical Industrial Institute, People's Republic of China) were used for separation. Analytical HPLC was preformed on a 
Waters 2690 separations module with an Alltech ELSD 2000 detector. Preparative HPLC was carried out on a Varian Pro-star solvent delivery module with a Varian Pro-star UV-Vis detector. LC/CD Analysis: HPLC separations were carried out with a Jasco HPLC system on Chiralcel IA column (5 $\mu \mathrm{m}$, $150 \times 4.6 \mathrm{~mm}$ ) for the enantiomers of 5 [separation factor $(\alpha) 2.29$, resolution $\left(R_{s}\right) 6.47$ and retention time $\left(\mathrm{t}_{\mathrm{r}}\right) 13.73 \mathrm{~min}$ for $(S)-\mathbf{5}, \mathrm{t}_{\mathrm{r}} 7.45 \mathrm{~min}$ for $(R)-5$ ], 7 [separation factor $(\alpha) 1.23$, resolution $\left(\mathrm{R}_{\mathrm{s}}\right) 1.87$ and retention time $\left(\mathrm{t}_{\mathrm{r}}\right) 7.79 \mathrm{~min}$ for $(S)-7, \mathrm{t}_{\mathrm{r}} 6.80 \mathrm{~min}$ for $(R)-7$ ] and 9 [separation factor $(\alpha) 2.21$, resolution $\left(\mathrm{R}_{\mathrm{s}}\right) 5.23$ and retention time $\left(\mathrm{t}_{\mathrm{r}}\right) 10.25 \min$ for $(S)-9, \mathrm{t}_{\mathrm{r}} 6.07 \min$ for $(R)-9$ ] eluted at 0.6 $\mathrm{mL} / \mathrm{min}$ with hexane/ethanol, 7:3. The LC/CD and LC/UV traces were recorded online at $270 \mathrm{~nm}$ with a Jasco J-810 CD spectropolarimeter equipped with a $1 \mathrm{~cm}$ pathlength HPLC flow cell and the chromatogram was zeroed right after the start of recording and hence relative absorbance was measured. The on-line CD and UV spectra (200-400 nm) were recorded simultaneously at the maxima of the UV peaks where the flow was stopped. CD ellipticity values $(\theta)$ were not corrected for concentration. For an LC-CD spectrum, three consecutive scans were recorded and averaged with $2 \mathrm{~nm}$ band width, $1 \mathrm{sec}$ response and standard sensitivity; noise reduction was not applied during the processing. The LC-CD spectrum of the eluent recorded in the same way was used as background. The UV-absorption trace was recorded as high tension voltage (HTV) and converted to absorbance. The concentration of the injected sample was set so that the HT value did not exceed $500 \mathrm{~V}$ in the HT channel down to $220 \mathrm{~nm}$.

\subsection{Plant material}

The whole plants of $P$. chinensis were collected in Xichou country, Yunnan Province, P. R. China, in April 2004 and identified by Jingui Shen, Shanghai Institute of Materia Medica, Chinese Academy of Sciences. A voucher specimen (No. 20050325) is deposited in the Herbarium of Shanghai Institute of Materia Medica.

\subsection{Extraction and isolation}

Powdered and air-dried whole plants of $P$. chinensis $(1.84 \mathrm{~kg})$ were extracted with EtOH- $\mathrm{H}_{2} \mathrm{O}(95: 5)$ in room temperature three times $(10 \mathrm{~L}$ each). After evaporation of EtOH in vacuo, the residue was dissolved in water $(6.0 \mathrm{~L})$ and then extracted with petroleum ether (PE), EtOAc and $n-\mathrm{BuOH}$. The PE fraction (93 g) was subjected to column chromatography (CC) over silica gel, eluted with a gradient of PE-EtOAc (100:1 to 0:1) to yield Fr.1 Fr.9. Fr.8 (2.0 g) was subjected to a silica gel column eluted 
with $\mathrm{CHCl}_{3}-\mathrm{MeOH}(100: 4)$ to yield subfractions Fr.8A Fr.8D. Fr.8A (400 mg) was repeatedly subjected to a silica gel column $\left(\mathrm{CHCl}_{3}-\mathrm{MeOH} 100: 4\right)$ and then a Sephadex LH-20 column $\left(\mathrm{CHCl}_{3}: \mathrm{MeOH} 1: 1\right)$ to give 1 (28 mg), and the residue (30 mg) was separated by Pre-HPLC $\left(\mathrm{CH}_{3} \mathrm{CN} / \mathrm{H}_{2} \mathrm{O} 25-55 \%, 15 \mathrm{ml} / \mathrm{min}, 290 \mathrm{~nm}\right)$ to give $3(7 \mathrm{mg})$ and $4(4 \mathrm{mg})$. Fr.9 (4.0 g) was subjected to a silica gel column $\left(\mathrm{CHCl}_{3}-\mathrm{MeOH} 100: 1\right.$ to $\left.100: 3\right)$ to yield subfraction Fr.9A Fr.9E. Fr.9A (800 mg) was separated by Pre-HPLC $\left(\mathrm{CH}_{3} \mathrm{CN} / \mathrm{H}_{2} \mathrm{O} 28-56 \%, 15 \mathrm{ml} / \mathrm{min}, 320 \mathrm{~nm}\right)$ and then purified by CC over Sephadex LH-20 (MeOH) to give 1 (16 mg) and 2 (23 mg). Fr.9B (400 mg) was separated by Pre-HPLC $\left(\mathrm{CH}_{3} \mathrm{CN} / \mathrm{H}_{2} \mathrm{O} 25-65 \%, 15 \mathrm{ml} / \mathrm{min}, 320 \mathrm{~nm}\right)$ to give $9(85 \mathrm{mg})$. Fr.9C (140 mg) was separated by Sephadex LH-20 $(\mathrm{MeOH})$ to yield 7 (60 mg). Fr.9D (430 mg) was separated by Pre-HPLC $\left(\mathrm{CH}_{3} \mathrm{CN} / \mathrm{H}_{2} \mathrm{O} 20-55 \%, 15 \mathrm{ml} / \mathrm{min}, 290 \mathrm{~nm}\right)$ and purified on a Sephadex LH-20 (MeOH) column to afford $5(28 \mathrm{mg}), \mathbf{6}(11 \mathrm{mg})$ and $\mathbf{8}(10 \mathrm{mg})$.

\subsection{Phochinenin G (1)}

Brown amorphous powder, $[\alpha]_{D}^{20}-0.005$ (c 0.315, MeOH). UV (MeOH) $\lambda_{\max } \mathrm{nm}(\log \varepsilon): 213(5.04)$, 279 (4.89), 296 (4.77). IR (KBr): $v_{\max } \mathrm{cm}^{-1}: 3423,1614,1438,1197,1159$. EIMS m/z (rel. int): 482 [M ] (100), 241 (12), 211 (3), 181 (2), 157 (2), 111 (2), 85 (4), 71 (4), 57 (4). HR-EIMS m/z: 482.1742 [M ] (calcd for $\left.\mathrm{C}_{30} \mathrm{H}_{26} \mathrm{O}_{6}, 482.1729\right) .{ }^{1} \mathrm{H}$ - and ${ }^{13} \mathrm{C}-\mathrm{NMR}$ : see Table1.

\subsection{Phochinenin H (2)}

Brown amorphous powder, UV (MeOH) $\lambda_{\max } \mathrm{nm}(\log \varepsilon): 211$ (4.67), 280 (4.51). IR (KBr): $v_{\max } \mathrm{cm}^{-1}$ : EIMS m/z (rel. int): 482 [M+] (100), 242 (19), 297 (6), 181 (4), 149 (4), 129 (4), 97 (4), 73 (6), 57 (8). HR-EIMS $m / z: 482.1733\left[\mathrm{M}^{+}\right]$(calcd for $\left.\mathrm{C}_{30} \mathrm{H}_{26} \mathrm{O}_{6}, 482.1729\right) .{ }^{1} \mathrm{H}-$ and ${ }^{13} \mathrm{C}-\mathrm{NMR}$ : see Table 1.

\subsection{Phochinenin I (3)}

Brown amorphous powder, UV (MeOH) $\lambda_{\max } \mathrm{nm}(\log \varepsilon): 201$ (4.68), 279 (4.22). IR (KBr): $v_{\max } \mathrm{cm}^{-1}$ : 3386, 1614, 1440, 1195, 1147. EIMS m/z (rel. int): 484 [M+] (100), 359 (8), 331 (6), 239 (4), 185 (8), 171 (14), 149 (22), 129 (20), 115 (70), 101 (18), 87 (12), 57 (56). HR-EIMS m/z: 484.1894 [M+] (calcd for $\left.\mathrm{C}_{30} \mathrm{H}_{28} \mathrm{O}_{6}, 484.1786\right)$. ${ }^{1} \mathrm{H}$ - and ${ }^{13} \mathrm{C}-\mathrm{NMR}$ : see Table 2. 


\subsection{Phochinenin J (4)}

Brown amorphous powder, UV (MeOH) $\lambda_{\max } \mathrm{nm}(\log \varepsilon)$ : 201 (4.74), 279 (4.29, 297 (4.12). IR (KBr): $v_{\max } \mathrm{cm}^{-1}: 3423,1614,1452,1197,1159$. EIMS m/z (rel. int): $484\left[\mathrm{M}^{+}\right]$(100), 377 (15), 345 (4), 239 (10), 185 (2), 129 (4), 115 (70), 97 (4), 73 (7), 57 (10). HR-EIMS m/z: 484.1879 [M+ ] (calcd for $\left.\mathrm{C}_{30} \mathrm{H}_{28} \mathrm{O}_{6}, 484.1786\right) .{ }^{1} \mathrm{H}$ - and ${ }^{13} \mathrm{C}-\mathrm{NMR}$ : see Table 2.

\subsection{Phochinenin K (5)}

Brown amorphous powder, UV (MeOH) $\lambda_{\max } \mathrm{nm}$ (log $\varepsilon$ ): 201 (4.83), 280 (4.33). IR (Film): $v_{\max } \mathrm{cm}^{-1}$ : 3392, 1606, 1557, 1457, 1197. EIMS m/z (rel. int): 484 [M+] (100), 346 (8), 242 (7), 211 (7), 197 (6), 149 (8), 107 (5), 77 (5), 57 (4). HR-EIMS m/z: $484.1871\left[\mathrm{M}^{+}\right]$(calcd for $\mathrm{C}_{30} \mathrm{H}_{28} \mathrm{O}_{6}, 484.1786$ ). ${ }^{1} \mathrm{H}$ - and

${ }^{13} \mathrm{C}-\mathrm{NMR}$ : Table 2. CD data recorded on-line in hexane/ethanol 7:3 for $(\mathrm{a} S)-5: \lambda_{\max }(\phi): 314(-2.3)$, 299sh (-2.2), 282 (-2.4), 268 (0.9), 257 (-0.8), 237 (4.0), 225 (-6.9). (aR)-5: 313sh (3.9), 301 (4.5), 282 (6.0), 270 (-0.5), 257 (1.6), 237 (-7.8), 227 (6.6).

\subsection{Phochinenin L (6)}

Brown amorphous powder, $[\alpha]_{D}^{20}+0.027(c 0.305, \mathrm{MeOH}) . \mathrm{UV}(\mathrm{MeOH}) \lambda_{\max } \mathrm{nm}(\log \varepsilon): 212$ (4.69), 281 (4.45), 297 (4.45). IR (KBr): $v_{\max } \mathrm{cm}^{-1}: 3417,1606,1459,1197,1157$. EIMS m/z (rel. int): 482 [M ] (100), 357 (8), 241 (4), 149 (12), 107 (8), 91 (8), 57 (15). HR-EIMS m/z: 482.1736 [M+] (calcd for $\left.\mathrm{C}_{30} \mathrm{H}_{26} \mathrm{O}_{6}, 482.1729\right) .{ }^{1} \mathrm{H}$ - and ${ }^{13} \mathrm{C}-\mathrm{NMR}$ : see Table 2.

\subsection{Gymconpin C (7)}

Brown amorphous powder, $[\alpha]_{D}^{20}+0.003(c 0.315, \mathrm{MeOH}) . \mathrm{CD}$ data recorded on-line in hexane/ethanol 7:3 for (aS)-7: $\lambda_{\max }(\phi): 307$ (8.7), 287sh (5.5), 271 (-9.1), $246(8.5), 219$ (-9.7). (aR)-7: 311 (-8.8), 286sh (-5.5), 269 (10.0), $243(-8.3), 222(9.8)$.

\subsection{Flavanthrin (9)}

Brown amorphous powder, $[\alpha]_{D}^{20} 0(c 0.380, \mathrm{MeOH}) . \mathrm{CD}$ data recorded on-line in hexane/ethanol 7:3 
for $(\mathrm{a} S)-9: \lambda_{\max }(\phi): 315$ (22.0), 303sh (13.6), 286sh (7.0), 268 (-18.9), 251sh (5.6), 236 (20.4), 221 (-25.1), 202 (-30.9). (aR)-9: 315 (-39.1), 305sh (-26.8), 285sh (-13.6), 269 (-34.6), 250sh (-12.8), 236 (-36.2), 220 (32.8), 204 (21.9).

\section{Computational Section}

PM3 and DFT calculations were run with Spartan'06, Wavefunction, Inc., Irvine CA, with default parameters and convergence criteria. ZINDO calculations were run with Gaussian'03W, Revision D.01, Gaussian, Inc., Pittsburgh PA.

Inversion barriers were calculated with geometry scans along the biaryl linkage torsional mode computed with semi-empirical method PM3, in the 0-360 deg interval with 10 deg steps. The input structures were the lowest-energy PM3 conformers (relative to the various possible arrangements of the dihydrophenantrene moieties). Scans were run twice (increasing and decreasing dihedrals) and the lowest energy value was kept for each dihedral angle. For compounds 3-5, reduced models were used with the (2'-hydroxyphenyl)ethyl chain replaced by an ethyl group. Inversion barriers amount to the PM3 energies of the structures with dihedral angles $\theta$ (between the linked aromatic planes) close to either 0 or $180 \mathrm{deg}$, relative to the absolute minimum energy found, in each case, with $\theta$ around \pm 90 deg. For the symmetrical dimer 9 , the two minima with $\theta \approx+90$ and $\theta \approx-90$ deg are isoergonic; for all other compounds, two minima differing by $<1 \mathrm{kcal} / \mathrm{mol}$ were found. For all compounds, the barriers calculated for $\theta \approx 0$ or $180 \mathrm{deg}$ differed by up to $15 \mathrm{kcal} / \mathrm{mol}$ (owing to the non-symmetrical patterns of substitution); the inversion barriers shown in Table 3 coincide with the less ergonic of the two possible pathways.

As input structures for ZINDO calculations, four (compounds 7 and 9) or two (reduced analog of 5) minimum-energy structures with (aS) absolute configuration ( $P$ chirality) were generated with PM3 and optimized with the DFT method at B3LYP/6-31G(d) level. For $\mathbf{7}$ and 9, the four structures are indicated as conf. 1 (aS, a'S, $\left.\mathrm{a}^{\prime \prime} S\right), 2\left(\mathrm{a} S, \mathrm{a}^{\prime} R, \mathrm{a}^{\prime \prime} R\right), 3\left(\mathrm{a} S, \mathrm{a}^{\prime} R, \mathrm{a}^{\prime \prime} S\right)$ and $4\left(\mathrm{a} S, \mathrm{a}^{\prime} S, \mathrm{a}^{\prime \prime} R\right)$, where the first chirality refers to the dihydrophenanthrene-dihydrophenanthrene linkage. The two structures for $\mathbf{5}$ are indicated as conf. $1\left(\mathrm{a} S, \mathrm{a}^{\prime} S\right)$ and $2\left(\mathrm{a} S, \mathrm{a}^{\prime} R\right)$. All hydroxy and methoxy groups were coplanar with aromatic rings and their conformation was not changed among the various conformers. Relative energies in $\mathrm{kcal} / \mathrm{mol}$ (and Boltzmann populations) of DFT structures were the following: compound 5, conf. 1 abs. min. (65\%), conf. $2+0.37$ (35\%); compound 7, conf. 1 abs. min. (34\%), conf. $2+0.22(23 \%)$, conf. $3+0.28$ (21\%), conf. $4+0.27$ (21\%); compound 9, conf. 1 abs. min. (40\%), conf. 2 and $3+0.35$ (22\% each), 
conf. $4+0.56(16 \%)$. Rotational strengths were computed for each structure with the semi-empirical ZINDO-S/CI method including all possible configuration interactions (full CI). CD spectra were generated as sum of Gaussians with 2500 and $4200 \mathrm{~cm}^{-1}$ half-height widths, using dipole-length computed rotational strengths. Average spectra were obtained by Boltzmann-weighting at $298 \mathrm{~K}$ using DFT energies.

\section{Acknowledgments}

Financial supports from the Ministry of Science and Technology (2004CB518902), and from the Shanghai Commission of Science and Technology (06DZ22028) are gratefully acknowledged. S. Antus and T. Kurtán thank the Hungarian Scientific Research Fund (OTKA) and National Office for Research and Technology (NKTH) for financial support (T-049436, NI-61336 and K-68429).

\section{References and Notes}

1. Majumder, P. L.; Banerjee, S., Tetrahedron, 1988, 44, 7303-7308.

2. Bai, L.; Kato, T.; Inoue, K.; Yamakit, M.; Takagi, S., Phytochemistry, 1991, 30, 2733-2735.

3. Majumder, P. L.; Pal, S.; Majumder, S., Phytochemistry, 1999, 50, 891-897.

4. Matsuda, H.; Morikawa, T.; Xie, H. H.; Yoshikawa, M., Planta Med., 2004, 70, 847-855.

5. Zhang, G. N.; Zhong, L. Y.; Annie Bligh, S.W.; Guo, Y. L.; Zhang, C. F.; Zhang, M.; Wang, Z. T.; Xu, L.S., Phytochemistry, 2005, 66, 1113-1120.

6. Guo, X. Y.; Wang, J.; Wang, N. L.; Kitanaka, S.; Liu, H. W.; Yao, X. S., Chem. Pharm. Bull., 2006, $54,21-25$.

7. Eliel, E. L.; Wilen, S. H., Stereochemistry of Organic Compounds, Wiley, New York, 1994, pp. $1142-1155$.

8. Rouge dos Santos, A.; Campbell Pinheiro, A.; Renno Sodero, A. C.; Sousa da Cunha, A.; Costa Padilha, M.; Mesquita de Sousa, P.; Paredes Fontes, S.; Paranho Veloso, M.; Manssour Fraga, C. A. Quimica Nova 2007, 30, 125-135.

9. Fletcher, S. P.; Dumur, F.; Pollard, M. M.; Feringa B. L., Science 2005, 310, 80-82.

10. Lloyd-Williams, P.; Giralt, E., Chem. Soc. Rev., 2001, 30, 145-157.

11. Jiangsu New Medical College. The Chinese Medicine Dictionary, Shanghai Science and Technology Press, Shanghai, P. R. China, 1997, 600-601.

12. Yao, S., Tang, C. P., Li, X. Q., Ye, Y., Helv. Chim. Acta, submitted. 
13. (a) Bao, J.; Wulff, W. D.; Dominy, J. B.; Fumo, M. J.; Grant, E. B.; Rob, A.C.; Whitcomb, M. C.; Yeung, S. M.; Ostrander, R. L.; Rheingold, A. L. J. Am. Chem. Soc., 1996, 118, 3392-3405; (b) Hattori, T.; Shimazumi, Y.; Goto, H.; Yamabe, O.; Morohashi, N.; Kawai, W.; Miyano, S., J. Org. Chem., 2003, 68, 2099-2108; (c) Hattori, T.; Sakurai, K.; Koike, N., Miyano, S., Goto, H., Ishiya, F., Harada, N., J. Am. Chem. Soc., 1998, 120, 9086-9087.

14. (a) Dai, J.; Krohn, K.; Flörke, U.; Draeger, S.; Schulz, B.; Kiss-Szikszai, A.; Antus, S. ; Kurtán, T.; Ree, T., Eur. J. Org. Chem, 2006, 15, 3498-3506; (b) Ferrari, J.; Terreaux, C.; Kurtán, T.; Kiss-Szikszai, A.; Antus, S.; Msonthi, J. D.; Hostettmann, K., Helv. Chim. Acta, 2003, 86, 2768-2778.

15. . Salvadori, P.; Di. Bari, L.; Pescitelli, G., HPLC-CD: Stereochemical Analysis at Work, in Circular Dichroism: Principles and Applications, ed. K. Nakanishi, N. Berova and R.W. Woody, Wiley-VCH, New York, 2nd ed., 2000, ch. 28, pp. 797-817.

16. Majumder, P.; Laha, S.; Datta, N., Phytochemistry, 1982, 21, 478-480.

17. Stewart, J. J. P., J. Comput. Chem. 1989, 10, 209-220 ; ibid., 221-264.

18. (a) Kranz, M.; Clark, T.; Schleyer, P. V. R., J. Org. Chem. 1993, 58, 3317-3325; (b) Spivey, A. C., Charbonneau, P. ; Fekner, T. ; Hochmuth, D. H. ; Maddaford, A. ; Malardier-Jugroot, C. ; Redgrave, A. J.; Whitehead, M. A., J. Org. Chem. 2001, 66, 7394-7401; (c) Di Bari, L.; Pescitelli, G.; Reginato, G. ; Salvadori P., Chirality 2001, 13, 548-555.

19. Harada, N.; Nakanishi, K., Circular Dichroic Spectroscopy - Exciton Coupling in Organic Stereochemistry, Oxford University Press, Oxford, 1983.

20. (a) Rashidi-Ranjbar, P.; Sandström, J., J. Mol. Struct., 1991, 246, 25-32. (b) Gawroński, J.; Grycz, P.; Kwit, M.; Rychlewska U., Chem. Eur. J., 2002, 8, 4210-4215.

21. (a) Ridley, J. E.; Zerner, M. C., J. Mol. Spectr., 1974, 50, 457-473; (b) Ridley, J.; Zerner, M., Theor. Chim. Acta, 1973, 32, 111-134.

22. Telfer, S.G.; Tajima, N.; Kuroda, R., J. Am. Chem. Soc., 2004, 126, 1408-1418, and references cited therein. 